\title{
Relation between Antigen Release and Immune Response of Oil Adjuvanted Vaccines in Chickens
}

\author{
Shin-ichi FUKANOKI, Kohei MATSUMOTO, Hajime MORI ${ }^{1)}$ and Reiji TAKEDA \\ Aburahi Laboratories Shionogi \& Co., Ltd. 1405 Gotanda, Koka-cho, Koka-gun, Shiga 520-3423 and ${ }^{1)}$ Department of Applied Biology, \\ Faculty of Textile Science, Kyoto Institute of Technology, Matsugasaki, Sakyo-ku, Kyoto 606-8585, Japan
}

(Received 1 December 1999/Accepted 1 February 2000)

ABSTRACT. The relationship between release properties of the model antigen, bovine serum albumin (BSA), from formulations in vitro and immune response after administration of various oil adjuvanted vaccines containing liquid paraffin was examined in chickens. The vaccine prepared at an hydrophile-lipophile-balance (HLB) number of 4.8 showed slower release of BSA and higher immune response on injected chickens than that with an HLB number of 6.0. Decreases of aqueous volume ratio in the formulation also led to slower release of BSA and higher immune response. The slower release rate of BSA showed higher ELISA antibody titer even at 20 weeks after vaccination. The ELISA antibody titer inversely was related to the constant release rate, $k$, calculated from the in vitro release test. The correlation coefficient was 0.863 . The immune response of oil adjuvanted vaccines containing Haemophilus paragallinarum agreed well with these results with BSA. Our results indicated that a stronger and more prolonged immune response of oil adjuvanted vaccines was achieved by slower release rate of antigen from the formulation. In addition, there was a good correlation between immune response and the value of $k$.-KEY WORDS: bovine serum albumin, controlled release, immune response, infectious coryza, oil adjuvanted vaccine.

J. Vet. Med. Sci. 62(6): 571-574, 2000

Prevention of infectious diseases through vaccination with oil adjuvanted vaccines is an efficient method of protecting poultry. Such vaccines prepared with liquid paraffin are readily adapted to many poultry diseases such as Newcastle disease, avian infectious bronchitis and infectious coryza (IC), and are widely used in the poultry industry $[1,5-8]$. These vaccines are emulsions consisting of an oil phase containing liquid paraffin and surfactants, and a water phase containing antigens. Herbert [3] suggested that oil adjuvanted vaccines may exert immune activity solely by the slow release of antigen. Further, Hilleman [4] and Gupta and Siber [2] noted that oil adjuvanted vaccines generally show special promise in providing long-term immunity. However, there have been no studies to clarify the mechanisms underlying such phenomena.

Here, we report the relationship between antigen release from formulations in vitro and immune response after administration of various oil adjuvanted vaccines of the model antigen, bovine serum albumin (BSA) or inactivated Haemophilus paragallinarum ( $\mathrm{Hpg}$ ) of the causal agent of IC, in chickens.

\section{MATERIALS AND METHODS}

Vaccine materials: The nonionic surfactants, sorbitan sesquioleate and polysorbate 80 , were kindly supplied by Kao Co. (Japan). Liquid paraffin was a gift from Witco Co. (U.S.A.). BSA (Fraction V) was purchased from Seikagaku Co. (Japan). The 221 strain of $H p g$, agglutination serovar A in IC, was used. The antigen of Hpg was prepared by reported method [9].

Chickens: Specific-pathogen-free (SPF) Aburahi chickens, 4-5 weeks old, were obtained from the SPF flock at Aburahi Laboratories, Shionogi \& Co., Ltd. (Shiga, Japan). The chickens were reared in isolation facilities maintained at a constant temperature.

Vaccine preparation: Oil adjuvanted vaccines containing antigen, BSA or Hpg in Dulbecco's phosphate buffered saline (-) at $\mathrm{pH} 7.4$ (PBS) as the aqueous phase and liquid paraffin as the oil phase, were prepared as follows. The aqueous phase was slowly added to the oil phase containing five vol\% nonionic surfactants, which were mixed with various ratios of sorbitan sesquioleate and polysorbate 80 . The aqueous-oil volume (A/O) ratios and hydrophilelipophile-balance (HLB) numbers of surfactants in formulation were $3 / 7,5 / 5,7 / 3$ and $4.8,6.0$, respectively. The HLB number was determined from proportionate weighted amounts of each surfactant: $a \times 3.7 /(a+b)+b \times$ 15.0/(a+b) (Eq.1), where "a" and "b" are amounts of sorbitan sesquioleate and polysorbate 80 , respectively. Then, components were sonicated using an ultrasonic disrupter UR-200P model (Tomy Seico Co., Japan) below $25^{\circ} \mathrm{C}$ for emulsification. A volume of $0.5 \mathrm{~m} l$ of vaccine contained $1.0 \mathrm{mg}$ of BSA or $10^{8}$ colony-forming units (CFU) of Hpg. As controls, aluminum hydroxide gel containing BSA or Hpg was prepared as described by Otsuki and Iritani [10] and Freund's incomplete adjuvant (FIA) containing Hpg was prepared with Adjuvant, Incomplete, Freund (Wako Pure Chemical Industries, Ltd., Japan).

Release tests: The release of BSA from vaccines was measured on the membranes in a vertical diffusion cell (Franz diffusion cell, Fig. 1). The cell had an effective area for diffusion of $0.85 \mathrm{~cm}^{2}$, and the receptor volume was about $14.0 \mathrm{~m} l$. The membrane was placed on the upper opening of the receptor compartment. The receptor solution was saline solution containing $20 \mathrm{vol} \%$ ethylene 


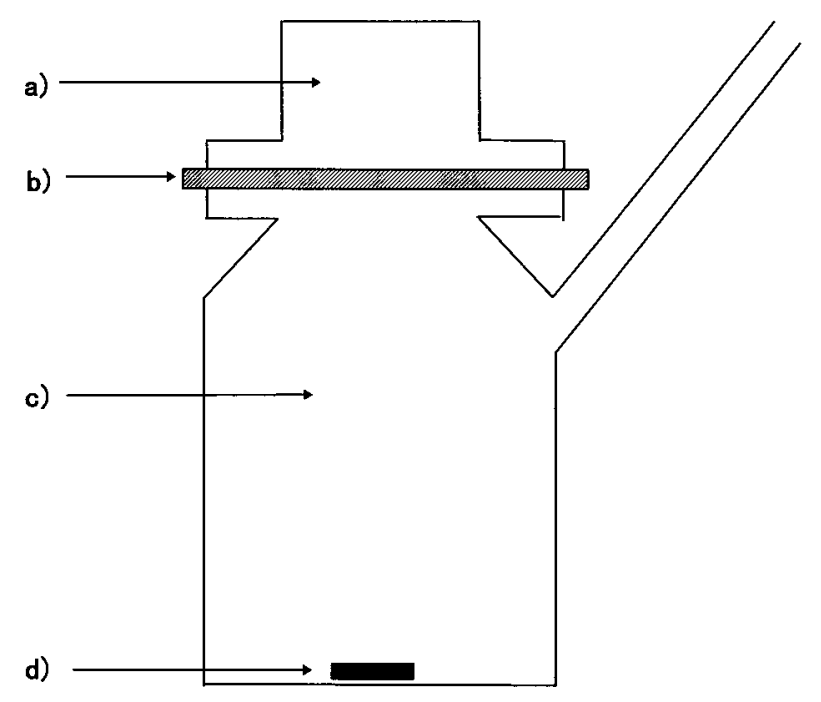

Fig. 1. Schematic of Franz diffusion cell assembly. a) cell cap (donor phase), b) membrane, c) receptor fluid chamber (receptor phase), d) stirring.

glycol, and was stirred during the experiment. The membrane was a $0.45 \mu \mathrm{m}$ polyvinilydenedifluoride filter (HV membrane, Nihon Millipore Co., Japan), which showed little effect on protein adsorption. All experiments were carried out at $40^{\circ} \mathrm{C}$. The concentrations of BSA in the receptor solution were measured by the Lowry method. Release rates from vaccines were determined from the equation $\mathrm{M}_{\mathrm{t}} / \mathrm{M}_{\infty}=k \cdot \mathrm{t}^{1 / 2}$ (Eq. 2), where $\mathrm{M}_{\mathrm{t}} / \mathrm{M}_{\infty}$ is the fraction of BSA released at time $\mathrm{t}, k$ is the release rate constant [11]. The values of $k$ were calculated from the slope of a plot based on Eq. 2.

Bioassays: Vaccines $(0.5 \mathrm{~m} / /$ chicken $)$ were injected into the thigh muscle of the chickens (5 birds/group). All chickens were bled periodically to determine ELISA antibody titers of BSA or hemagglutination-inhibition (HI) antibody titers of $\mathrm{Hpg}$ [9] after vaccination. In the case of $\mathrm{HI}$ serology, four hemagglutination units were used as a standard procedure in microtiter plates. Each antibody titer was obtained from the geometric mean titer. Aluminum hydroxide gel containing BSA or Hpg and Freund's incomplete adjuvant (FIA) containing $\mathrm{Hpg}$ were injected in the same manner as oil adjuvanted vaccines as controls. Statistical significance was assessed by using Student's paired $t$ test. A $p$ value of $<0.05$ was considered significant.

ELISA antibody titer to BSA: Ninety-six well immunoplates (Falcon 3072, Becton Dickinson Co., France) were coated overnight at $4^{\circ} \mathrm{C}$ with $100 \mu \mathrm{l} / \mathrm{well}$ of $2 \%$ BSA in carbonate-bicarbonate buffer at $\mathrm{pH}$ 9.6. The plates were washed with PBS containing $0.1 \%$ Tween 20 as a washing buffer. After adding blocking buffer of PBS containing 3\% skim milk, the plates were washed. And the test serum (100 $\mu l /$ well) was added to the wells. In the assay, the test serum diluted from 1:100 to 1:102400 was added and incubated for two hours at room temperature. The plates were washed, and a 1:2500 dilution of peroxidase-conjugated sheep anti-chicken IgG was added to each well and incubated for one hour at room temperature. After washing, $o$ phenylene diamine dihydrochloride [0.02\% in citrate-phosphate buffer, 0.004 vol $\% \mathrm{H}_{2} \mathrm{O}_{2}$ ] was used as a substrate. After five minutes, the reaction was stopped by the addition of $100 \mu \mathrm{l} / \mathrm{well}$ of $2 \mathrm{~N}$ sulfuric acid. Absorbency in each well was determined with a microplate autoreader (Multiskan ${ }^{\circledR}$ Bichromatic; Labosystems Japan Co., Japan) at $492 \mathrm{~nm}$.

\section{RESULTS}

Antigen release from oil adjuvanted vaccines: Figure 2 shows the effects of HLB numbers on the release of BSA from vaccines prepared at an $\mathrm{A} / \mathrm{O}$ ratio of $3 / 7$. Release of BSA from the oil adjuvanted vaccine at an HLB number of 4.8 was slower than that at an HLB of 6.0. Figure 3 shows the effect of $\mathrm{A} / \mathrm{O}$ ratios on the release of $\mathrm{BSA}$ from vaccines. The release rate of BSA decreased with decreasing volume of aqueous phase.

Effects of $H L B$ number and $A / O$ ratio on anti-BSA antibody: Figure 4 shows ELISA antibody titers of BSA after administration of the oil adjuvanted vaccines at HLB numbers of 4.8 or 6.0. The vaccine at an HLB number of 4.8 induced greater ELISA antibody titers than that an HLB of 6.0. The vaccine also maintained higher ELISA antibody titer even at 20 weeks after vaccination $(p<0.05)$. Figure 5 shows ELISA antibody titers of BSA after administration of the vaccines at $\mathrm{A} / \mathrm{O}$ ratios of $3 / 7,5 / 5$ and $7 / 3$. ELISA antibody titer after vaccination increased with increasing oil volume ratio in the formulation. And ELISA antibody titer of the vaccine at an $\mathrm{A} / \mathrm{O}$ ratio of $3 / 7$ was the highest even at 20 weeks after vaccination $(p<0.05)$. Figure 6 shows the relationship between release rate constants, $k$, and ELISA antibody titers at 20 weeks after vaccination. A good correlation was found between these factors, with a correlation coefficient of 0.863 .

Effects of A/O ratio on anti-IC antibody: Figure 7 shows $\mathrm{HI}$ antibody titers to IC at three weeks after vaccination of the oil adjuvanted vaccines, at an HLB number of 4.8, and various $\mathrm{A} / \mathrm{O}$ ratios. The oil adjuvanted vaccine at an $\mathrm{A} / \mathrm{O}$ ratio of $3 / 7$ showed the highest $\mathrm{HI}$ antibody titer of all the vaccines examined $(p<0.05)$.

Comparison of anti-IC antibody between formulations: $\mathrm{HI}$ antibody titer of the oil adjuvanted vaccine prepared with an HLB number of 4.8 and an A/O ratio of 3/7 was compared with that of other formulations containing FIA or aluminum hydroxide gel (Table 1). The experimental oil adjuvanted vaccine induced the greatest $\mathrm{HI}$ antibody titer of all the vaccines examined, and had an HI antibody titer of 23 even at 10 weeks after vaccination.

\section{DISCUSSION}

The relationship between antigen release from oil adjuvanted vaccines and immune response after 


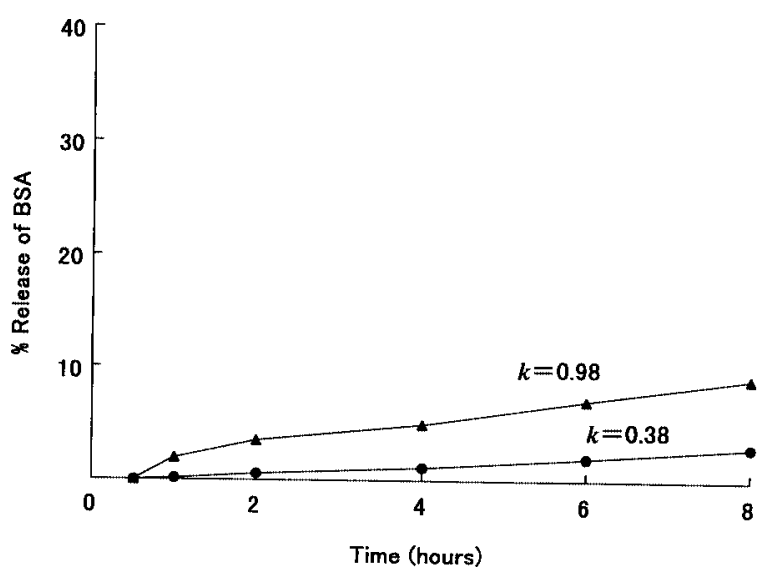

Fig. 2. Release of BSA from oil adjuvanted vaccines at HLB numbers of 4.8 or 6.0. : HLB 4.8, $\boldsymbol{\Delta}$ : HLB 6.0. Each point represents the mean of 3 experiments. The value of $k$ is the release rate constant.

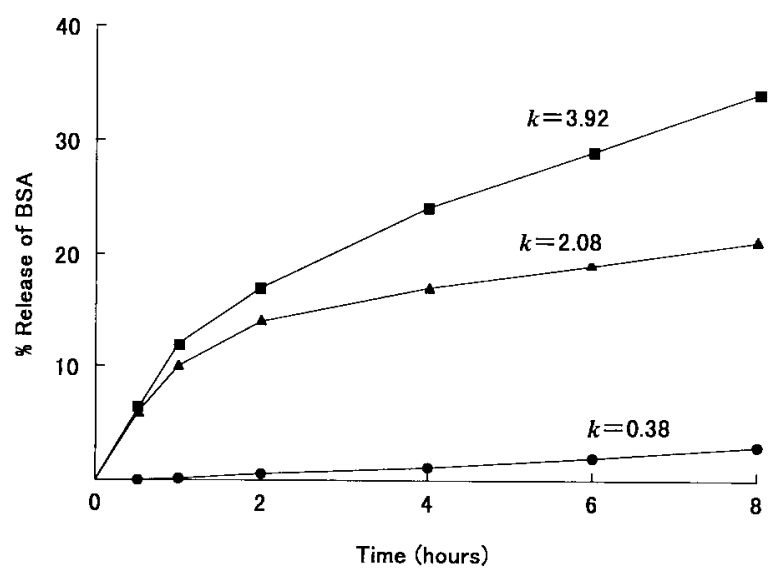

Fig. 3. Release of BSA from oil adjuvanted vaccines at various $\mathrm{A} / \mathrm{O}$ ratios. $: \mathrm{A} / \mathrm{O}=3 / 7, \boldsymbol{\Delta}: \mathrm{A} / \mathrm{O}=5 / 5, \boldsymbol{\square}: \mathrm{A} / \mathrm{O}=7 / 3$. Each point represents the mean of 3 experiments. The value of $k$ is the release rate constant.

administration of them was investigated in chickens. The release rate constant, $k$, of the vaccine at an HLB number of 4.8 was smaller than that at an HLB of 6.0 (Fig. 2). Furthermore, the vaccine of small $k$ showed a stronger and more prolonged immune response (Fig. 4). Decreases in the aqueous volume ratio in the formulation reduced release rate of BSA and increased ELISA antibody titer (Figs. 3, 5). Antigen release rate was dependent on HLB number and/or $\mathrm{A} / \mathrm{O}$ ratio in the formulation. It was also found that $k$ was inversely related to the ELISA antibody titer after vaccination (Fig. 6). These results indicated that a smaller value of $k$ could adequately account for the high and sustained adjuvant activity.

In the case of oil adjuvanted vaccines containing $\mathrm{Hpg}$ at an HLB number of 4.8, HI antibody titer of three weeks

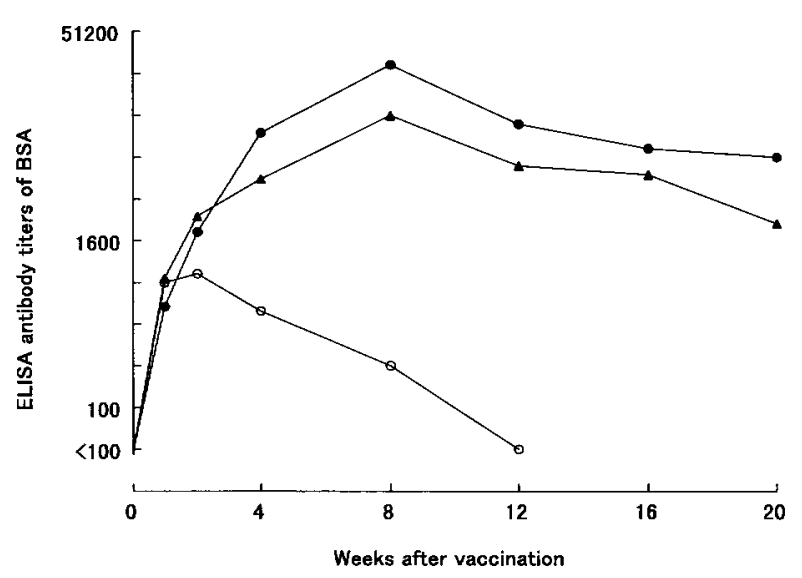

Fig. 4. ELISA antibody titers of BSA after vaccination of oil adjuvanted vaccines at HLB numbers of 4.8 or 6.0. : HLB 4.8, $\boldsymbol{\Delta}$ : HLB 6.0, $\bigcirc$ : Aluminum hydroxide gel. Each point represents the geometric mean titer of 5 birds.

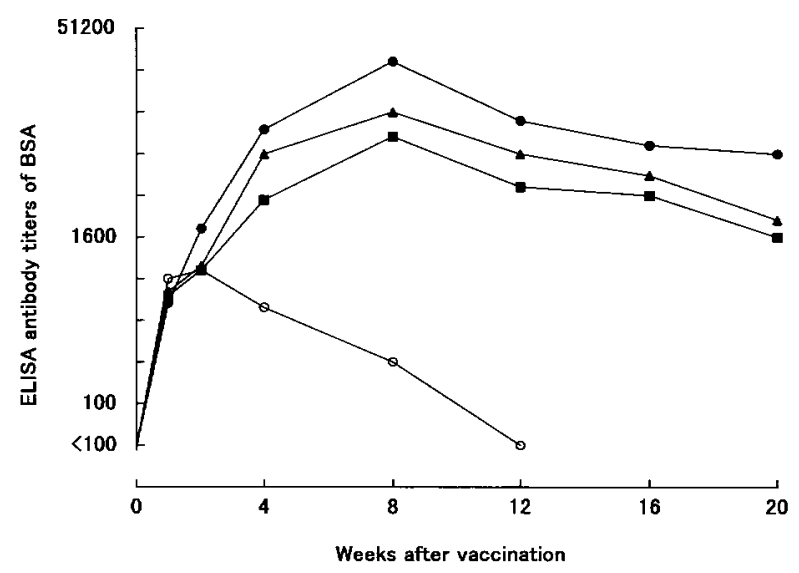

Fig. 5. ELISA antibody titers of BSA after vaccination with oil adjuvanted vaccines at various $\mathrm{A} / \mathrm{O}$ ratios. $\mathrm{A} / \mathrm{O}=3 / 7, \boldsymbol{\Delta}$ : $\mathrm{A} / \mathrm{O}=5 / 5, \boldsymbol{\square}: \mathrm{A} / \mathrm{O}=7 / 3, \bigcirc:$ Aluminum hydroxide gel. Each point represents the geometric mean titer of 5 birds.

after vaccination increased with decreasing aqueous volume ratio (Fig.7). The vaccine prepared with a low aqueous volume ratio, i.e. an $\mathrm{A} / \mathrm{O}$ ratio of $3 / 7$, maintained high $\mathrm{HI}$ antibody titer even at 10 weeks after vaccination (Table 1). Thus, the results of Hpg also agreed well with those of BSA. Reid and Blackall [7] reported that the immune response of an inactivated IC oil adjuvanted vaccine was less than that of an aluminum hydroxide gel vaccine. They observed no strong immune response by a vaccine containing liquid paraffin as an adjuvant. However, it was possible in our present experiments to produce a strong and prolonged immune response to IC by the oil adjuvanted vaccine prepared with an HLB number of 4.8 and an $\mathrm{A} / \mathrm{O}$ ratio of 3/7. Thus, slow release of antigen from the formulation seems to be important for the immune 


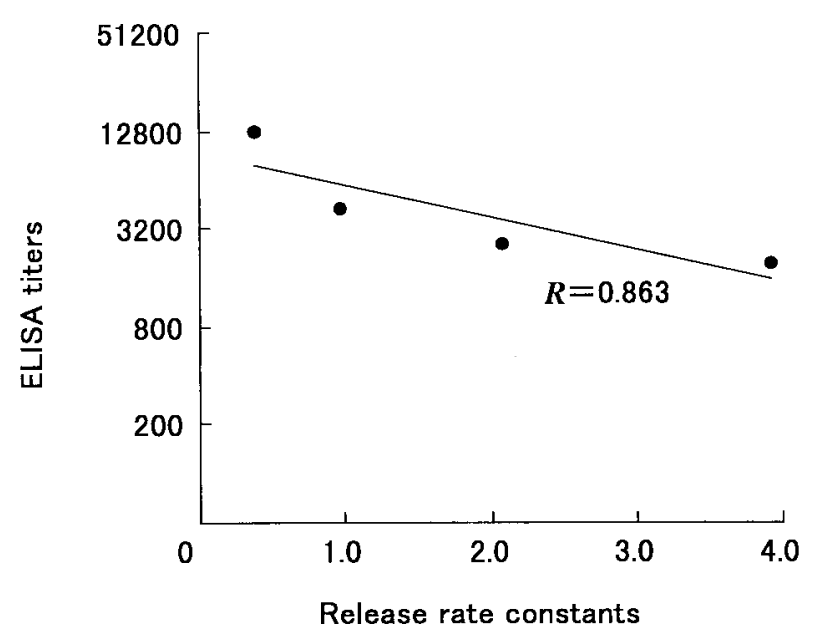

Fig. 6. Relationship between the release rate constants and ELISA antibody titers at 20 weeks after vaccination with oil adjuvanted vaccines containing BSA. The value of $R$ is the correlation coefficient.



Fig. 7. HI antibody titers at 3 weeks after vaccination with oil adjuvanted vaccines, HLB number of 4.8 , containing $\mathrm{Hpg}$ at various $\mathrm{A} / \mathrm{O}$ ratios.

Table 1. Comparison of Hi htiters after vaccination with different types of formulations containing $\mathrm{Hpg}$

\begin{tabular}{lcccccc}
\hline & \multicolumn{5}{c}{ HI antibody titers } \\
\cline { 2 - 7 } & $2 \mathrm{wk}^{\mathrm{b})}$ & $3 \mathrm{wk}$ & $4 \mathrm{wk}$ & $5 \mathrm{wk}$ & $8 \mathrm{wk}$ & $10 \mathrm{wk}$ \\
\hline Oil adjuvanted vaccine $^{\mathrm{a})}$ & 7 & 256 & 676 & 256 & 91 & 23 \\
Freund's incomplete adjuvant vaccine $_{\text {Aluminum hydroxide gel vaccine }}$ & $<2$ & $<2$ & 5 & 8 & 5 & $\mathrm{NT}^{\mathrm{c}}$ \\
Alu & 9 & 112 & 112 & 28 & $\mathrm{NT}$ & $\mathrm{NT}$ \\
\hline
\end{tabular}

a) HLB: 4.8 and A/O ratio: 3/7, b) Weeks after vaccination, c) Not tested. Each point represents the geometric mean titer of 5 birds examined.

response.

Consequently, the strong and prolonged immune response of oil adjuvanted vaccine was found to be achieved by slow release of antigen. In addition, the immune response and release ratio constant, $k$, showed a good correlation. So, we assumed that the release of antigen, as determined by in vitro release test, would be an important factor determining the immune response and thus should be taken into account when preparing a new vaccine.

\section{REFERENCES}

1. Ganne, V., Eloit, A., Laval, A., Adam, M. and Trouve, G. 1994. Enhancement of the efficacy of a replication-defective adenovirus-vectored vaccine by the addition of oil adjuvants. Vaccine 12: 1190-1196.

2. Gupta, R.K. and Siber, G.R. 1995. Adjuvants for human vaccines-current status, problems and future prospects. Vaccine 13: $1263-1276$.

3. Herbert, W.J. 1968. The mode of action of mineral-oil emulsion adjuvants on antibody production in mice. Immunology 14: 301-318.

4. Hilleman, M.R. 1966. Critical appraisal of emulsified oil adjuvants applied to viral vaccines. Prog. Med. Virol. 8: 131182.

5. Jacobs, A.A.C., Cuenen, W. and Storm, P.K. 1992. Efficacy of a trivalent Haemophilus paragallinarum vaccine compared to bivalent vaccines. Vet. Microbiol. 32: 43-49.

6. Peleg, B.A., Samina, I. and Brenner, J. 1993. Immunization of chickens with live Newcastle disease vaccine adjuvanted with oil. Vaccine 11: 1074-1076.

7. Reid, G.G. and Blackall, P.J. 1986. Comparison of adjuvants for an inactivated infectious coryza vaccine. Avian Dis. 31: $59-63$.

8. Stone, H.D. 1991. The preparation and efficacy of manually emuisified Newcastle disease oil-emulsion vaccines. Avian Dis. 35: 8-16.

9. Yamaguchi, T., Takigami, S., Iritani, Y. and Hayasi, Y. 1990. Characterization and use of monoclonal antibodies to identify Haemophilus paragallinarum serovars. Avian Dis. 34: 52-57.

10. Otsuki, K. and Iritani, Y. 1973. Preparation and immunological response to a new mixed vaccine composed of inactivated Newcastle disease virus, inactivated infectious bronchitis virus, and inactivated Hemophilus gallinarum. Avian Dis. 18: 297-304.

11. Lee, P.I. 1985. Kinetics of drug release from hydrogel matrices. J. Control. Release 2: 277-288. 